\title{
Effectiveness of Etching by Three Acids on the Morphological and Chemical Features of Dentin Tissue
}

\author{
Naji Kharouf ${ }^{1}$, Davide Mancino², Asâd Naji-Amrani ${ }^{3}$, Ammar Eid $^{4}$, Youssef Haikel ${ }^{5}$, Joseph Hemmerle ${ }^{6}$
}

\begin{abstract}
Aim: The purpose of this study was to evaluate the microscopic and chemical effects of phosphoric acid gel, phosphoric acid liquid, and polyacrylic acid application for 15 seconds (s) on coronal dentin.

Materials and methods: Twelve extracted teeth were selected. Three etching acids were used to prepare the dentin surfaces. Scanning electron microscopy (SEM) and X-ray energy dispersive spectroscopy (EDX) were used to analyze the chemical and morphological changes of the dentinal surfaces, including the depth of demineralization. Collected data were statistically analyzed by the one-way analysis of variance test.

Results: Dentin etched with phosphoric acid gel or liquid showed greater peritubular dentin dissolution, including complete removal of the smear layer. In addition, there were many silica particles on the dentin etched by phosphoric acid gel $37 \%$. The dentin that was etched with $25 \%$ polyacrylic acid for 15 seconds showed no smear layer removal. Chemical analysis (EDX) showed that dental surfaces etched with phosphoric acid liquid $37 \%$ for 15 seconds showed the strongest mineral dissolution at the calcium surface, with a calcium content of $5.25 \%$. On the other hand, EDX analysis of the dental surface etched with $25 \%$ polyacrylic acid showed more surface enrichment in calcium (17.19\%).

Conclusion: Although phosphoric acid (gel or liquid) 37\% cleans the dental surface, phosphoric acid gel precipitates silica particles on the etched dentin surface. These particles cannot be removed by rinsing off this acid. The application of polyacrylic acid for 15 seconds does not noticeably demineralized dentin, nor remove the smear layer.

Clinical significance: The clinician should use phosphoric acid (gel or liquid) to clean dental surfaces to prepare them for the bonding process. The low demineralizing effects of the polyacrylic acid permits its use near the pulp.

Keywords: Dentin demineralization, Phosphoric acid, Polyacrylic acid.

The Journal of Contemporary Dental Practice (2019): 10.5005/jp-journals-10024-2626
\end{abstract}

\section{INTRODUCTION}

Dentin is the substance located under the enamel, surrounding the pulp chamber and root canals. The dentin microstructure is composed of dentinal tubules that outwardly emerge into the dentin structure from the pulp to the outer cementum or the enamel boundary. Peritubular dentin and intertubular dentin are enriched with collagen fibers. There are variations between the size, quantity, and wall thickness of dentin tubules from the outside to the inside. ' Dentinal tubules diameter ranges from $2.5 \mu \mathrm{m}$ (near the pulp) to less than $1 \mu \mathrm{m}$ (near the enamel), enamel adjacent surfaces bounded by a highly mineralized cuff of peritubular dentin. The intertubular dentin is composed of collagen fibers directed mainly perpendicular to dentin tubules, loaded with apatite crystallites. ${ }^{2}$

Important clinical considerations have been considered when etching the dentin, based on its composition. Dentin composition is roughly $70 \%$ mineral, $20 \%$ organic, and $10 \%$ water. $^{3}$

The recent improvements in materials have made bonding to dentin more effective. Metal alloys, ceramics, and composites require special surface processing for effective bonding. A smear layer (an adherent layer of debris) usually covers tooth surfaces requiring bonding, hence requiring sufficient cleaning in the preparation process. This smear layer can form on dentin during procedural preparation with burs and hand instruments. It contains crushed hydroxyapatite and fractured collagen. Bacteria and saliva may also contaminate this layer. ${ }^{4}$

The dentin smear layer could be described as a disturbed film of organic and hydroxyapatite particles, generally less than $2 \mathrm{~mm}$ thick. It has been generally accepted that the composition
1,2,5 Department of Endodontics, Faculty of Dental Medicine, Strasbourg University, Strasbourg, France; INSERM UMR_S 1121, Biomaterials and Bioengineering, Strasbourg, France

3,6INSERM UMR_S 1121, Biomaterials and Bioengineering, Strasbourg, France

${ }^{4}$ Department of Endodontics, Faculty of Dental Medicine, Damascus University, Syria

Corresponding Author: Naji Kharouf, Department of Endodontics, Faculty of Dental Medicin, Strasbourg University, Strasbourg, France; INSERM UMR_S 1121, Biomaterials and Bioengineering, Strasbourg, France, Phone: +33 667522841, e-mail: dentistenajikharouf@ gmail.com

How to cite this article: Kharouf N, Mancino D, et al. Effectiveness of Etching by Three Acids on the Morphological and Chemical Features of Dentin Tissue. J Contemp Dent Pract 2019;20(8):915-919.

Source of support: Nil

Conflict of interest: None

of the smear layer is a mixture of partly denatured collagen and mineral. ${ }^{5-7}$ The most common disadvantage of the smear layer is that it diminishes bonding to underlying dentin. ${ }^{4}$ Durability and effective bonding between the dentin and an adhesive resin are essential for the longevity of restorations. ${ }^{8}$ Previous studies have shown that the efficiency of dentin adhesives depends on the removal of the smear layer and the generation of the resin-dentin inter-diffusional area. ${ }^{9}$ Acidic conditioners are most commonly used to treat the dentin surface. They eliminate the smear layer, open dentinal tubules, expose collagen fibrils, and alter dentin

(c) The Author(s). 2019 Open Access This article is distributed under the terms of the Creative Commons Attribution 4.0 International License (https://creativecommons. org/licenses/by-nc/4.0/), which permits unrestricted use, distribution, and non-commercial reproduction in any medium, provided you give appropriate credit to the original author(s) and the source, provide a link to the Creative Commons license, and indicate if changes were made. The Creative Commons Public Domain Dedication waiver (http://creativecommons.org/publicdomain/zero/1.0/) applies to the data made available in this article, unless otherwise stated. 
permeability and wetness. ${ }^{10,11}$ The bonding operation of adhesive systems to the dentin surface is essentially micromechanical. ${ }^{12}$

In operative dentistry, typically phosphoric acid is used in both liquid and gel forms. Multiple studies show that dentin collagen fibrils that are exposed to phosphoric acid have conformational modifications. ${ }^{13}$

Polyacrylic acid etching is commonly employed in restorative dentistry to lightly etch dentin in preparation for glass-ionomer cement placement. ${ }^{14}$

Increased bonding efficiency depends on the elimination of cutting debris, successful cavity preparation, partial demineralization, and the creation of microporosities. ${ }^{15}$

The chemical structure of teeth provides more information about mechanical strength, especially the weight percent of calcium and phosphorus. Hydroxyapatite contains calcium and phosphorus critical for mineral strengthening teeth. ${ }^{16}$

In this paper, the effects of three acids on dentin etching structure were determined using SEM. The chemical composition of the dentin was further characterized using EDX. ${ }^{17,18}$

The primary objectives of this paper were the investigation of the microstructure, and chemical composition of coronal dentin etched with different acids, including examining the depth of dentin penetration for the three types of acid preparations. The null hypothesis was that there were no differences in the microstructure and chemical composition of the dentin structure after etching procedure among three different acids.

\section{Materials and Methods}

Twelve recently extracted, caries-free, human maxillary third molars were selected. This in vitro study was conducted at the Faculty of Dental Medicine of Strasbourg University. These teeth were washed with physiological serum and stored at $4^{\circ} \mathrm{C}$ in ethanol $70 \%$. Two sections were made perpendicular to the longitudinal axis of the crown tooth to obtain a single coronal dentin wafer of $2 \mathrm{~mm}$ thickness, polished with a P320-grit silicon carbide paper (Escil, Chassieu, France) for 60 seconds under water cooling to obtain a similar surface to that obtained with dental diamond bur drill. ${ }^{19}$

We divided the extracted teeth into four groups (GI, GII, GIII, and GIV), and each group consisted of three teeth. In the first group $(\mathrm{Gl})$, the dentin of these teeth was considered as a control group without treatment. In the second group (GII), the dentin was etched with phosphoric acid gel 37\% (ITENA, Paris, France) for 15 seconds. In the third group (GIII), the dentin was etched with phosphoric acid liquid 37\% (Sigma-Aldrich, Darmstadt, Germany) for 15 seconds. The fourth group (GIV) was etched with polyacrylic acid $25 \%$ using a micro-brush for 15 seconds. A pH meter (CyberScan ph/Ion 510, Waltham, Massachusetts, USA) was used to measure hydrogen ion activity of the three acids (GII, GIII, and GIV).

After acid treatment, all etched teeth were rinsed with water for 15 seconds and dehydrated in a graded ethanol series. The samples were sputter-coated with gold-palladium alloys (20/80) using a HUMMER JR sputtering device (Technics, CA, USA). Analysis with a Quanta 250 FEG scanning electron microscope (FEl Company, Eindhoven, The Netherlands) functioning with an accelerating voltage of the electrons of $10 \mathrm{kV}$ was used to observe surfaces of these coated specimens. The chemical analysis of specimens was performed with an EDAX Octane Plus spectrometer (AMETEK Materials Analysis Division, Mahwah, NJ 07430, USA) for 10 zones of $443 \mu^{2}$ for each sample. After that, a mesiodistal section was made in the middle of every dentin wafer with wire saw (Walter EBNER, Le Locle, Switzerland) and prepared for chemical analysis (EDX). Compositional analysis was performed using the linescanning mode of the micro-EDX in order to investigate the depth of demineralization in dentin thickness. All the samples were considered for the SEM and EDX analyses.

Data analysis was performed with Sigma Plot (release 11.2, Systat Software, Inc., San Jose, CA, USA). The one-way analysis of variance test was applied in the comparisons of the mean calcium (Ca) mass percentages for the dentin etched with the different acids. In all tests, a statistical significance level of $\alpha=0.05$ was adopted.

\section{Results}

\section{pH Meter Measurements}

$\mathrm{A} \mathrm{pH}$ values were obtained and controlled by a $\mathrm{pH}$ meter where phosphoric acid gel had $\mathrm{pH}$ of 0.2 , phosphoric acid liquid had $\mathrm{pH}$ of -0.14 , and polyacrylic acid had $\mathrm{pH}$ of 1.6 .

\section{SEM Observation Dentin Surfaces}

Dentin that was not treated showed a thick smear layer covering the dentinal surface (Fig. 1A). After etching, dentin morphology exhibited specific characteristics and demineralization degrees according to the acid employed. Dentin which was etched with a phosphoric acid gel of $37 \%$ for 15 seconds showed larger open tubule diameters as a result of dissolution of peritubular dentin (Fig. 1B). Dentin that was etched with phosphoric acid liquid 37\% showed a well-defined circumference of dentin tubules without a smear layer, and a higher presence of collagen fibers (Fig. 1C). Otherwise, samples that were etched with polyacrylic acid showed microcracks, rough, and scaly surfaces without opening-up tubules (Fig. 1D). A layer of residual material was observed on the surface etched by the phosphoric acid gel 37\% (Fig. 2).

\section{EDX Analysis}

The analysis of the superficial layer of dentin-etched samples (GII) of remaining material after EDX showed that particles attached to the dentin surface, as observed by SEM images (Fig. 2) were silica particles. This silica was used to thicken the acid gel. We did not find these small silica particles on the dentin surfaces etched with non-gel-based acids (GIII, GIV).

The chemical composition of dentin etched with the three acids exhibits Ca mass percentages (means and standard deviations) reported in Table 1, which shows also the composition of the non-treated dentin (GI). The one-way analysis of variance reveals a statistically significant difference between the means corresponding to the different acids and the absence of etching. In all pairwise comparisons, the test leads to $p<0.001$, except for the pair (GII, GIII) where $p=0.023$. Therefore, the null hypothesis of insensitivity of the dentin to the etching is rejected $(p<\alpha)$. Specifically in the dentin etched by phosphoric acid gel or liquid (GII or GIII, respectively) as well as by polyacrylic acid (GIV), the Ca contents were $(6.95 \pm 1.64) \%$, $(5.25 \pm 1.17) \%$ and $(17.19 \pm 3.54) \%$, respectively. All these values are significantly different between them and differ significantly from the non-treated dentin (GI) where the Ca content is $(23.48 \pm 2.45) \%$.

The analysis by the line-scanning mode for the depth of demineralization showed that the phosphoric acid gel (GII) had the most demineralization depth of $6 \mu \mathrm{m}$ in dentin thickness comparing with phosphoric acid liquid $(4.5 \mu \mathrm{m})$ and polyacrylic acid $(4.5 \mu \mathrm{m})$. 

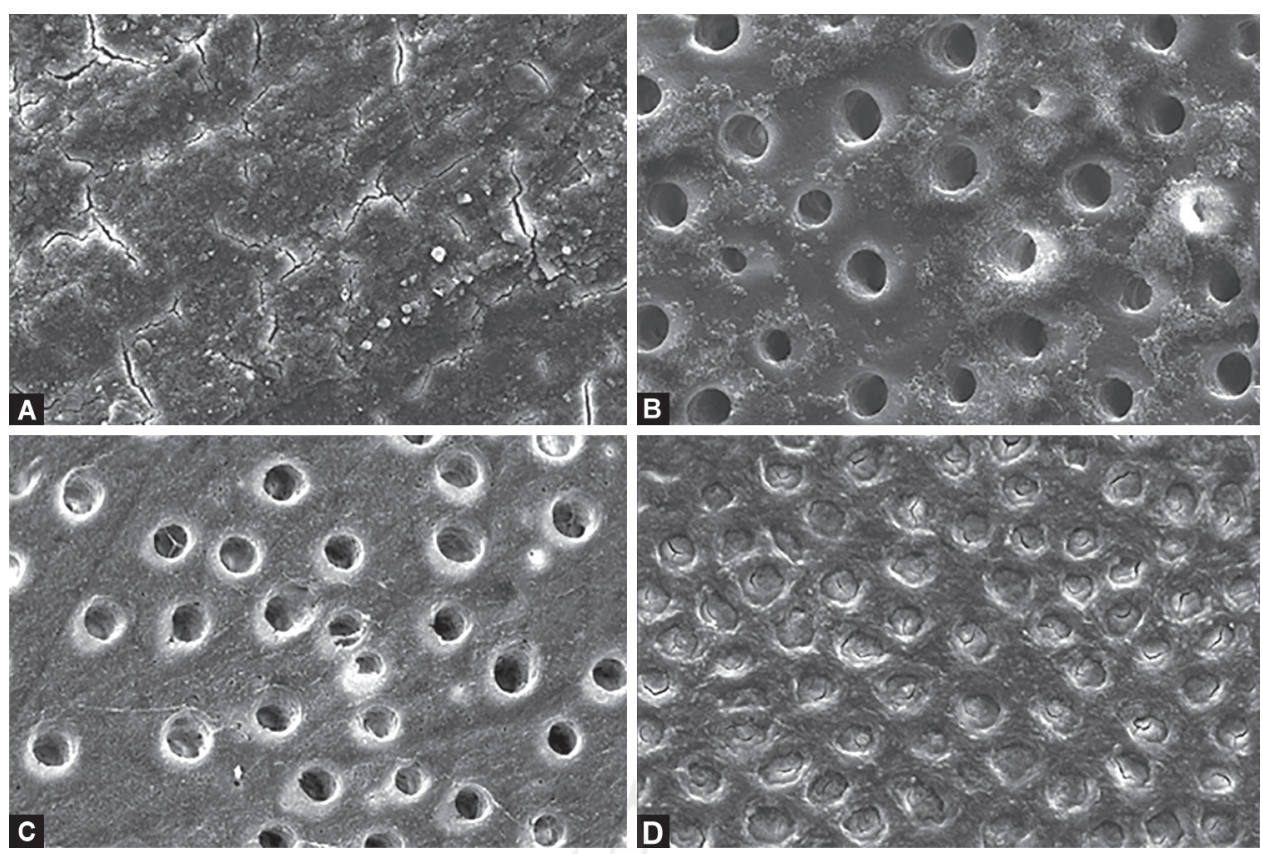

Figs 1A to D: (A) SEM micrograph of dentin surface showing complete closure of the dentin tubule entry points by the smear layer; (B) Dentin surface etched with phosphoric acid gel $37 \%$ for 15 seconds. Open tubules on the surface with numerous silica particles; (C) Dentin surface etched with phosphoric acid liquid 37\% for 15 seconds. Open tubules on the surface with no silica particles present; (D) SEM micrograph of dentin surface etched with polyacrylic acid 25\%. Dentin tubules are visible, but not open, because of plugging from the smear layer covering dentin surfaces

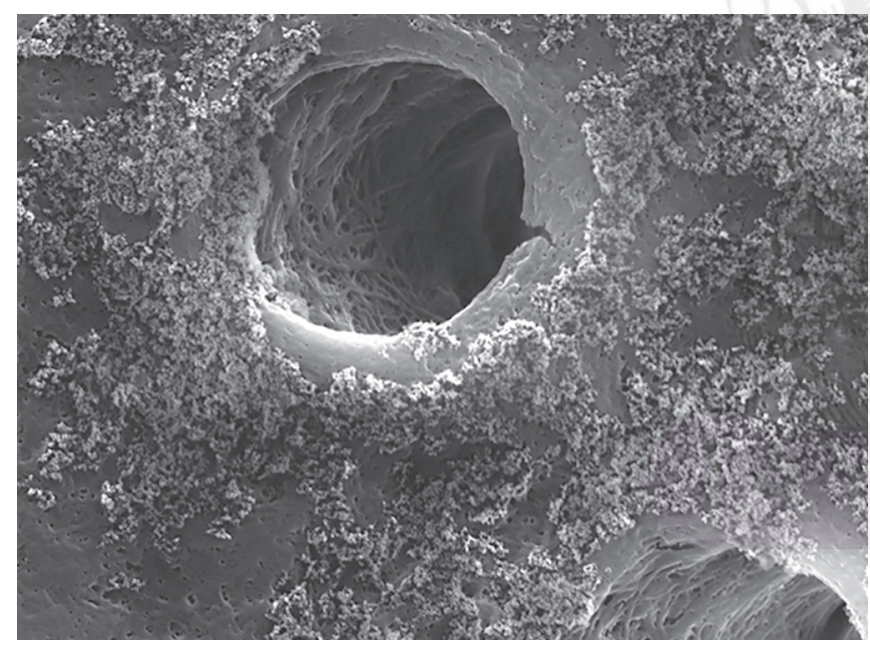

Fig. 2: Higher magnification of dentin etched with phosphoric acid gel $37 \%$. Amounts of small debris (silica) are clearly observed

Table 1: Mass percentage of calcium (mean \pm standard deviation) on dentin surfaces etched with the three acids (groups II-IV), and for the control group $(\mathrm{Gl})$, measured in 10 zones of $443 \mu \mathrm{m}^{2}$ each

\begin{tabular}{lllll}
\hline & Group I & Group II & Group III & Group IV \\
\hline Ca mass (\%) & $23.48 \pm 2.45$ & $6.95 \pm 1.64$ & $5.25 \pm 1.17$ & $17.19 \pm 3.54$ \\
\hline
\end{tabular}

\section{Discussion}

Tooth preparation processes create important changes in dentin thickness, and density leading to different resin bonding results. ${ }^{20}$

For this reason, smear layers produced clinically with abrasive papers (P320-grit silicon carbide paper) were used to produce a denser smear layer in the present study. ${ }^{21}$
Dentin surfaces covered with a smear layer (GI) typical of those produced by abrasive papers were used as the fully mineralized dentin control, as they have the highest mineral content, along with closed dentin tubules.

Hydroxyapatite crystals in the smear layer are loosely compressed, interconnected debris delicately attached to the tooth structure. ${ }^{6}$

In the light of these findings, the acid conditioning of dentin must be considered as an essential step in achieving clean dentin, and consequently reliable bond strength. ${ }^{22}$ The thick smear layer on the dentin surface that forms during diamond bur use is removed by etching with phosphoric acid liquid and gel $37 \%$ for 15 seconds (Figs $1 B$ and C). SEM imaging indicated that dentin surfaces exhibited different morphological appearances: dissolution of both the smear layer and peritubular dentin, removal of smear plug, and exposure of collagen fibers. Similar results have been found for dentin and enamel by Perdigão ${ }^{23}$ and Tao et al., ${ }^{24}$ who noted that the use of $30-40 \%$ phosphoric acid remove the smear layer and smear plugs. All groups showed the presence of a demineralized collagen matrix, except for the non-etched dentin control group (GI) and when polyacrylic acid was used (GIV). The application of phosphoric acid for 15 seconds revealed demineralized collagen matrix by SEM and dissolutions of both smear plugs and peritubular dentin. There was no obvious breakup in dentin. The demineralized collagen matrix was only seen after etching with phosphoric acid, but not after polyacrylic acid. Therefore, conditioning the dentin with polyacrylic acid removes the smear layer structure except for smear plugs. Moreover, this acid partially demineralizes the dentin surface in accordance with Summitt. ${ }^{25}$ This confirms our study which shows the poor efficacy of polyacrylic acid in etching through relatively denser smear layers. ${ }^{26}$ Peutzfeldt reported that the dentinal tubules were opened totally and cleared to a considerable depth by using $25 \%$ polyacrylic acid solution for 30 seconds. ${ }^{27}$ 
The silica particles attached to the dentin surface in GII (Figs 1B and 2) can have an influence on the dentin bonding effectiveness, as a result impairing bonding infiltration. The silica used to thicken the acid gel is not entirely removed by water rinsing. ${ }^{28}$ Although $10-20$ $\mathrm{nm}$ silica particles were found, they were small enough to infiltrate into the dentin surface. The use of acids to demineralize dentin results in collagen becoming positively charged, hence showing a higher affinity to the negative silica nanoparticles. ${ }^{29}$ The liquid acids used in (GIII, GIV) were silica free; hence no silica remnants were found on dentin surfaces (Figs $1 C$ and D).

After morphological observation, chemical analysis using EDX linked morphological change with altered chemical compositions at the dentin surface.

The chemical composition of non-treated samples (GI) contained C, N, O, P, and Ca. After acid treatments, Ca mass has been decreased in three groups (GII, GIII, and GIV), apparently due to dissolution of hydroxyapatite on the dentin surface.

Since calcium phosphates are dissolved with low $\mathrm{pH}$ phosphoric acid (gel and liquid), these can be rinsed from the dentin surface. The demineralization process impairs the mineral composition of the dentin and, as a consequence, less hydroxyapatite crystals and calcium are present. The use of phosphoric acid liquid resulted in a lower Ca residual on the dentinal surface (Table 1) due to its better wettability and lower surface energy compared with the gel formulation. Our hypothesis is that the fluid can easily infiltrate into the dentinal tubules and dissolve more hydroxyapatite.

The depth of the demineralization created by the acid gel was greater $(6 \mu \mathrm{m})$. This finding for the depth of the demineralization is in line with the result of Perdigão et al. ${ }^{30}$ The depth of the demineralization created by the liquid phosphoric acid was $4.5 \mu \mathrm{m}$, or with polyacrylic acid was $4.5 \mu \mathrm{m}$, due to the form of the gel acid that can remain attached to the surface of dentin longer than the liquid acid. Although studies showed that acidic conditioners increase intratubular permeability by removing smear plugs and the mineral content of peritubular dentin to a depth of approximately 2-7 $\mu \mathrm{m}^{31,32}$

Oyarzún et al. ${ }^{28}$ found that phosphoric acid liquid increases intertubular permeability more than phosphoric acid gel by elimination not only of the mineral content but also the organic backbone that supports the spatial organization of hydroxyapatite in peritubular dentin.

Consequently, the $\mathrm{pH}$ and the form of the etching acid are critical. These parameters affect the strength and depth of demineralization. Eliades et al. ${ }^{13}$ noted that the acid composition, $\mathrm{pH}$, osmolality, and viscosity produced by thickening agents have been identified as the major factors governing the dentinconditioner interactions.

Our study shows that the degree of demineralization on the dentin surface was proportional to the $\mathrm{pH}$ level of acids. EDX analysis revealed a low calcium content on the dentin surface after the application of a low $\mathrm{pH}$ phosphoric acid liquid.

Although phosphoric acid liquid has lower $\mathrm{pH}$ level, phosphoric acid gel with higher $\mathrm{pH}$ level has a deeper etching effect on dentin than liquid acid. We conclude this is because gel acids remain on the dental surface longer than liquid acids, and this contact time has a greater effect on the dentin depth than the $\mathrm{pH}$.

This study has limitations. The distinction between smear layer and the rough dentin surfaces in GIV was difficult. In addition, obliquely oriented tubules hinder the observation of the presence of debris within apparently opened tubules in some SEM graphs.

\section{Conclusion}

According to our results, the morphological and chemical composition of dentin varied in accordance with etching-acid formations. The characteristics specific for each treatment will have clinical implications for adhesive retention. It is important to determine the most important demineralization criterion for optimal bonding, including the depth and the degree of demineralization. Future studies are required to investigate and evaluate the effects of small particles of silica on the bonding retention.

\section{Clinical Significance}

The clinician should use phosphoric acid (gel or liquid) to clean a dental surface and prepare it for the bonding process. The gel acid has advantages over the liquid acid, including longer retention on the tooth surface and better control during application. The weak demineralization effects of polyacrylic acid permit its use near the pulp.

\section{Author Contribution}

Naji Kharouf and Davide Mancino contributed equally to this work.

\section{References}

1. Garberoglio R, Brannstrom M. Scanning electron microscopic investigation of human dentinal tubules. Arch Oral Biol 1976;21(6):355362. DOI: 10.1016/S0003-9969(76)80003-9.

2. Marshall GW Jr, Marshall SJ, et al. The dentin substrate: structure and properties related to bonding. J Dent 1997 Nov;25(6):441-458. DOI: 10.1016/S0300-5712(96)00065-6.

3. Goldberg $M$, Kulkarni $A B$, et al. Dentin: structure, composition and mineralization. Front Biosci (Elite Ed) 2011 Jan;3:711-735. DOI: 10.2741/e281.

4. Gwinnett AJ. Smear layer: morphological considerations. Oper Dent Suppl 1984;3:2-12.

5. Eick JD. Smear layer-Materials surface. Proc Finn Dent Soc 1992; 88(Suppl 1):225-242.

6. Eick JD, Wilko RA, et al. Scanning electron microscopy and electron microprobe analysis of cut tooth surfaces. J Dent Res 1970 NovDec;49(Suppl. 6):1359-1368. DOI: 10.1177/00220345700490063601.

7. Pashley DH. Smear layer: overview of structure and function. Proc Finn Dent Soc 1992;88(Suppl 1):215-224.

8. Bonfante EA, Pegoraro LF, et al. SEM observation of the bond integrity of fiber-reinforced composite posts cemented into root canals. Dental Materials 2008 Apr;24(4):483-491. DOI: 10.1016/j.dental.2007.04.010.

9. $\mathrm{Gu} \mathrm{XH}, \mathrm{Mao} \mathrm{CY}$, et al. Does endodontic post space irrigation affect smear layer removal and bonding effectiveness? Eur J Oral Sci 2009 Oct;117(5):597-603. DOI: 10.1111/j.1600-0722.2009.00661.x.

10. Bertolotti RL. Conditioning of the dentin substrate. Oper Dent 1992(Suppl 5):131-136.

11. Erickson RL. Surface interactions of dentin adhesive materials. Oper Dent 1992;17(Suppl 5):81-94.

12. Pashley DH, Tay FR, et al. State of the art etch-and-rinse adhesives. Dental Materials 2011;27:1-16. DOI: 10.1016/j.dental.2010.10.016.

13. Eliades $G$, Palaghias $G$, et al. Effect of acidic conditioners on dentin morphology, molecular composition and collagen conformation in situ. Dent Mater 1997 Jan;13(1):24-33. DOI: 10.1016/S0109-5641(97) 80005-X.

14. Ozcan S, Seseogullari-Dirihan R, et al. Effect of polyacrylic acid on dentin protease activities. Dent Mater 2015 Aug;31(8):901-906. DOI: 10.1016/j.dental.2015.04.018.

15. Hajizadeh $\mathrm{H}$, Ghavamnasiri M, et al. Effect of different conditioning protocols on the adhesion of a glass ionomer cement to dentin. J Contemp Dent Pract 2009 Jul;10(4):9-16. DOI: 10.5005/jcdp-10-4-9. 
16. Jeng YR, Lin TT, et al. Human enamel rod presents anisotropic nanotribological properties. J Mech Behav Biomed Mater 2011 May;4(4):515-522. DOI: 10.1016/j.jmbbm.2010.12.002.

17. Stape THS, Wik P, et al. Selective dentin etching: a potential method to improve bonding effectiveness of universal adhesives. J Mech Behav Biomed Mater 2018 Oct;86:14-22. DOI: 10.1016/j.jmbbm.2018.06.015.

18. Moyaho-Bernal M, Contreras-Bulnes R, et al. Morphological and chemical changes in human deciduous dentin after phosphoric acid, self-etching adhesive and Er:YAG laser conditioning. Microsc Res Tech 2018 May;81(5):494-501. DOI: 10.1002/jemt.23003.

19. Stape THS, WikP, et al. Selective dentin etching: a potential method to improve bonding effectiveness of universal adhesives. J Mech Behav Biomed Mater 2018 Oct;86:14-22. DOI: 10.1016/j.jmbbm.2018.06.015.

20. Sattabanasuk V, Vachiramon V, et al. Resin-dentin bond strength as related to different surface preparation methods. J Dent 2007 Jun;35(6):467-475. DOI: 10.1016/j.jdent.2007.01.002.

21. Armstrong S, Breschi L, et al. Academy of Dental Materials guidance on in vitro testing of dental composite bonding effectiveness to dentin/enamel using micro-tensile bond strength ( $\mu \mathrm{TBS}$ ) approach. Dent Mater 2017 Feb;33(2):133-143. DOI: 10.1016/j.dental.2016.11.015.

22. Zhang $L$, Huang $L$, et al. Effect of post-space treatment on retention of fiber posts in different root regions using two self-etching systems. Eur J Oral Sci 2008 Jun;116(3):280-286. DOI: 10.1111/j.16000722.2008.00536.x.

23. Perdigão J. Dentin bonding-variables related to the clinical situation and the substrate treatment. Dent Mater 2010 Feb;26(2):e24-e37. DOI: 10.1016/j.dental.2009.11.149.
24. Tao L, Pashely DH, et al. Effect of different types of smear layers on dentin and enamel shear bond strengths. Dent Mater 1988;4:208-216. DOI: 10.1016/S0109-5641(88)80066-6.

25. Summitt JB, Santos JD. Fundamentals of operative dentistry: acontemporary approach, 3rd ed. Chicago: Quintessence; 2006.

26. El-Askary FS, Nassif MS, et al. Shear bond strength of glass-ionomer adhesive to dentin: effect of smear layer thickness and different dentin conditioners. J Adhes Dent 2008 Dec;10(6):471-479.

27. Peutzfeldt A, Asmussen E. Effect of polyacrylic acid treatment of dentin on adhesion of glass ionomer cement. Acta Odontol Scand 1990 Oct;48(5):337-341. DOI: 10.3109/00016359009033626.

28. Oyarzún A, Rathkamp H, et al. Immunohistochemical and ultrastructural evaluation of the effects of phosphoric acid etching on dentin proteoglycans. Eur J Oral Sci 2000 Dec;108(6):546-554. DOI: $10.1034 / j .1600-0722.2000 .00912 . x$.

29. Besinis A, van Noort $R$, et al. Infiltration of demineralized dentin with silica and hydroxyapatite nanoparticles. Dent Mater 2012 Sep;28(9):1012-1023. DOI: 10.1016/j.dental.2012.05.007.

30. Perdigão J, May KN Jr, et al. The effect of depth of dentin demineralization on bond strengths and morphology of the hybrid layer. Oper Dent 2000 May-Jun;25(3):186-194.

31. Eick JD, Gwinnett AJ, et al. Current concepts on adhesion to dentin. Crit Rev Oral Biol Med 1997;8(3):306-335. DOI: 10.1177/ 10454411970080030501.

32. Pashley DH, Carvalho RM. Dentine permeability and dentine adhesion. J Dent 1997 Sep;25(5):355-372. DOI: 10.1016/S03005712(96)00057-7. 